\title{
Paul Vidal de la Blache (1845-1918)
}

géographe français (fondateur de la géographie moderne)

(1902)

\section{"Les conditions géographi- ques des faits sociaux"}

Un document produit en version numérique par Michel Côté, bénévole, aménagiste de la MRC de Bécancour

Courriel: cote_mic@yahoo.ca

Dans le cadre de la collection: "Les classiques des sciences sociales"

Site web: http://classiques.uqac.ca/

Une collection développée en collaboration avec la Bibliothèque

Paul-Émile-Boulet de l'Université du Québec à Chicoutimi

Site web: http://bibliotheque.uqac.ca/ 
Un document produit en version numérique par M. Michel Côté, bénévole, aménagiste de la MRC de Bécancour

Courriel: cote_mic@yahoo.ca à partir de :

Paul Vidal de la Blache (1845-1918)

"Les conditions géographiques des faits sociaux"

Un article publié dans la revue Annales de géographie, vol. XI, 1902, pp. 13-23.

Polices de caractères utilisée :

Pour le texte: Times New Roman, 14 points.

Pour les citations : Times New Roman 12 points.

Pour les notes de bas de page : Times New Roman, 12 points.

Édition électronique réalisée avec le traitement de textes Microsoft Word 2004 pour Macintosh.

Mise en page sur papier format : LETTRE (US letter), $8.5^{\prime \prime} \mathrm{x}$ $11 ")$

Édition numérique réalisée le 8 mars 2006 à Chicoutimi, Ville de Saguenay, province de Québec, Canada. 
Marcel J. Paul Vidal de la Blache (1845-1918)

"Les conditions géographiques des faits sociaux"

Une édition électronique réalisée à partir de l'article de Paul Vidal de la Blache (1845-1918) "Un article publié dans la revue Annales de géographie, vol. XI, 1902, pp. 13-23.

L'étude des conditions géographiques des faits sociaux est une question dont l'importance ne trouverait guère de contradicteurs. Mais je n'étonnerai personne en disant que cette importance est plutôt pressentie que connue. Tant sur l'objet précis de la recherche que sur la méthode à suivre, les idées manquent de clarté; les preuves d'une certaine confusion se rencontrent fréquemment dans les conversations et dans les écrits. Je vais donc essayer de m'expliquer ici sur ces deux points; et pour cela, je partirai d'abord d'exemples très simples.

Il est assurément facile de saisir des cas de corrélation intime entre un fait géographique et un fait social. La contiguïté de deux régions, plaine et montagne, où l'ordre des travaux n'est pas le même, où les récoltent mûrissent à des dates différentes, rend disponibles des travailleurs qui vont louer périodiquement leurs bras. La présence d'une grande ville fait naître à ses portes des cultures spéciales, associées à des habitudes également spéciales, celles des maraîchers ou des hortillons. L'existence très localisée d'un produit de première nécessité peut engendrer des conséquences sociales et politiques. Tout le monde sait quelle importance historique a eue le commerce du sel en Bavière, en Lorraine, en Franconie et ailleurs; à quels mouvements d'échanges il a donné lieu sur certains points du Sahara. Source de richesse et de 
puissance pour ses détenteurs, la possession de ce bien provoquait des conflits, créait des relations, contribuait souvent à former des villes.

Ces rapports sont intéressants; l'historien et l'économiste se plaisent à les noter. Mais, si curieux qu'il puisse être d'assembler des faits de ce genre, on peut se demander s'ils constituent un objet de science, s'il est possible de fonder sur eux une recherche systématique et méthodique. Non sans doute, si on les envisage isolément, comme des incidents et des particularités. Mais n'en sera-t-il pas autrement si on s'élève à une notion plus compréhensive et plus haute ? N'y a-t-il pas un plan général dans lequel rentrent ces exemples, ou d'autres semblables, de phénomènes sociaux ?

Avant de répondre à ces questions, je crois utile de rappeler que, dans cet ordre de faits, nos moyens de recherche se sont notablement accrus depuis un demi-siècle. Les progrès de la connaissance du globe et la colonisation nous ont mis en rapport avec un nombre de plus en plus grand de sociétés humaines à des degrés très inégaux de développement. On a étudié leurs genres de vie; l'attention s'est portée d'une façon méthodique sur leurs moyens de nourriture, le vêtement, l'habitation, les instruments, les armes, bref sur l'ensemble d'objets dans lequel s'Expriment les habitudes, les dispositions et les préférences de chaque groupe.

On a constaté aussi bien des diversités, dont le principe, comme on peut s'en convaincre, est surtout dans les différences de matériaux fournis par la nature ambiante. Mais on est arrivé aussi par la comparaison à constater que par-dessus les variantes locales il y avait des formes d'existence, des modes de civilisation embrassant de grandes étendues et de nombreuses collections d'êtres humains.

Ces diverses formes de civilisation se manifestent d'une façon concrètes par les objets qu'elles créent à leur usage, ce qu'on a pris l'habitude d'appeler leur matériel ethnographique. Le mot fait songer involontairement à ces vitrines de musée où sont assemblés armes, parures, défroques et ustensiles de tribus sauvages; et il n'y a pas à se plaindre de cette association d'idées, si elle a pour effet d'enfoncer en 
nous cette notion que la civilisation la plus rudimentaire comme la plus raffinée est digne d'attention, qu'elle a sa place, si modeste soitelle, dans les archives de l'humanité. Mais le mot, dans ce qu'il implique de signalement caractéristique, est tout aussi applicable à de grands types de civilisation. Il y a dans la nourriture, le vêtement, le mobilier, les constructions, l'Art médical dont le Chinois fait usage, un fonds commun emprunté à la nature inorganique ou vivante, sur lequel son ingéniosité s'est exercée, et qui reste comme sa signature de peuple. Je dirai même que ces docks, ces élévateurs, ces puissantes machines avec lesquelles l'Américain manie les quantités et les masses, sont dans leur genre des documents ethnographiques, des signes caractéristiques de sa civilisation. En cela, comme dans les objets dont le Négritien ou la Malais ont emprunté la matière et la forme à la nature végétale qui les entoure, se manifeste un effort d'invention et de perfectionnement en rapport avec un certain milieu.

Il est aisé de juger quel précieux renfort ces expressions diverses d'industrie humaine apportent à l'étude géographique des faits sociaux. Les institutions et les mœurs n'ont pas figure matérielle; mais ce sont choses étroitement liées aux objets que l'homme a façonnés sous l'influence du régime social auquel est adaptée sa vie. Ces objets reflètent des habitudes qui dérivent de l'état social ou qui l'inspirent. Nous gagnons ainsi pour nos recherches un degré qui nous met de plain-pied avec elles; et grâce à l'universalité des documents fournis, nous sommes mieux en situation de comprendre comment, non dans un cas particulier, mais d'une façon générale et coordonnée, les faits géographiques qu'impriment sur la vie sociale.

\section{II}

La cause qui, d'après nous, introduit le plus de différences entre les sociétés, est la position. Suivant qu'une contrée est vouée à l'isolement, ou qu'elle est ouverte, au contraire, aux courants de vie générale, les rapports des hommes entre eux sont tout autres. C'est l'éternelle antithèse qui frappait Thucydide, lorsqu'il opposait en Grèce les peuples parvenus à ce degré de civilisation qu'exprimait le mot (...), et ceux qui pratiquait encore la manière de vivre archaïque. Ces tribus restées primitives, il les trouverait encore là où il les signale. Ces communautés, retranchées dans leurs conditions tradition- 
nelles d'existence, ont en général la vie dure. C'est, si l'on y songe, un sujet de réflexion autant que de surprise, que de voir autour de notre Méditerranée tant de peuples, dont plusieurs hautement doués, dont le régime social porte encore l'empreinte de l'isolement. Là se perpétue la vie de clan et de tribu, où l'autorité politique n'Excède pas le cercle où elle peut s'exercer d'une façon matérielle et directe, où l'habitude persiste d'aller armé, où s'éternisent les guerres de vendetta entre familles ou tribus. Le mépris d tout ce qui est étranger est inhérent à cette forme de société. La personne de l'étranger n'est protégée que par des rites d'hospitalité dont l'efficacité cesse à la porte de l'hôte, ou par l'usage de contrats personnels.

La montagne, la forêt, surtout la forêt tropicale avec ses impénétrables lacis de lianes et de troncs pourris, de grandes étendues à franchir soit à travers les continents, soit à travers les mers : voilà ce qui a longtemps maintenu et qui maintient encore un grand nombre de groupes humains à distance les uns des autres. Il y avait, sans remonter au delà de quarante ans, dans le centre de l'Afrique, de nombreuses populations chez lesquelles n'avait jamais pénétré un Arabe, ni un Européen. Dans la zone africaine de forêts tropicales, c'est le village qui est l'unité, chacun formant un petit monde à part, dans la clairière qu'il cultive. Et pourtant, parmi ces groupes vivant dans un état qui paraît si rudimentaire, il en est qui ont su tirer un ingénieux parti des matériaux fournis par la nature ambiante, et dont le matériel ethnographique, tel qu'on peut l'étudier au Musée de Berlin, ne manque ni de richesse, ni de variété. Tant il est vrai qu'on est bien en présence d'une forme de société arrêtée, se développant à sa manière, douée d'une impulsion propre ! Nos officiers ont pu constater même, au Soudan, que le long de la lisière septentrionale de la forêt ce type de village isolé pousse comme des rejetons; dans les lambeaux détachés de la forêt se gîtent des villages, qui ont soin de barricader par des obstacles l'accès de leur clairière.

Il est rare pourtant que l'isolement et l'organisme social strictement renfermé en lui-même soient absolus. Une tribu peut en avoir une autre ou d'autres dans sa clientèle. Les sof ou partis qui divisent chaque ksar dans le Sud-Ouest de l'Algérie, ont des amis et des ennemis dans d'autres ksour. Dans l'Afrique centrale elle-même on connaît, par les récits des explorateurs, ce curieux exemple de parasi- 
tisme social, rappelant certains sociétés animales, qui s'est établi entre le pygmée chasseur et le villageois cultivateur de la zone forestière tropicale, et dans lequel chacune des deux parties trouve occasion d'un échange utile.

Nous venons de parler de l'isolement qui résulte des conditions naturelles; mais il y a aussi l'isolement voulu, méthodique, cartésien pourrait-on dire. C'est celui que recherchent des civilisés pour s'émanciper des entraves d'une société gênante, et réaliser telle forme sociale ou religieuse. Ainsi firent en 1847 ceux qui allèrent chercher dans les solitudes du Lac Salé la liberté de s'organiser à leur guise, qui leur était refusée dans les États de l'Est. Dans les vallées les plus reculées de l'Altaï, sur les frontières de Chine, ou bien même au delà du cercle polaire dans les éclaircies de la grande forêt sibérienne, des colonies de raskolniks ont vécu ainsi, isolées, ignorées; et c'est seulement bien tard que la colonisation actuelle, qui les relance aujourd'hui dans leur retraite, a révélé leur existence. Citerons-nous de petits villages d'anabaptistes qui se sont créé une existence à part dans quelques vallées retirées autour du Donon ? Il ne serait pas tout à fait juste de dénier à ces échappées d'affranchissement toute portée générale. À quel autre sentiment obéirent les Puritains qui vinrent au XVIe siècle aborder aux côtes du Massachusetts ? Et ne suffirait-il pas de feuilleter Hérodote pour trouver dans la colonisation ancienne des exemples analogues ? Nous touchons ainsi à une série de faits intéressants dont on peut dire, il est vrai, que les progrès des communications rendent chaque jour le renouvellement plus difficile. Il n'est peut-être pas sans inconvénient que le champ disponible pour ces expériences se restreigne sans cesse. Ces phénomènes de géographie sociale étaient susceptibles d'engendrer une série de conséquences dont l'originalité n'est souvent qu'un régal pour le sociologue, mais qui, en certains cas, ont pu servir de ferment à des sociétés naissantes.

De même que la position, les traits physiques d'une contrée s'impriment profondément dans l'état social. La contiguïté de la steppe pastorale et des terres de culture, de l'oasis et du désert, comme celle de la plaine et de la montagne, est une cause de rapports dont la portée politique et économique ne saurait être méconnue sans inconvénient. Nous l'avons appris à nos dépens, en Algérie. Habitués par des livres et des théories dogmatiques à opposer, en les cantonnant 
dans des domaines respectifs, l'agriculteur et le pasteur, comme deux formes de vie sans pénétration réciproque, on n'est arrivé qu'assez lentement à concevoir la véritable nature de leurs relations mutuelles. Tel est pourtant le cas qui se présente non seulement sur la lisière du Sahara, mais dans une grande partie de l'Afrique et de l'Asie. L'exemple de la région des ksour, que nous citions tout à l'heure, permet de prendre ces rapports sur le vif. Dans son ksar semblable au vieil oppidum italiote, entre ses murailles percées de rares portes et dont l'enceinte déjà étroite se subdivise souvent encore elle-même en quartiers fermés, le cultivateur sédentaire met en sûreté les récoltes des jardins que vivifient aux abords immédiats ses rigoles d'irrigation. Il est cultivateur et artisan; des tissus et des instruments, la plupart fabriqués par les femmes, s'emmagasinent avec les grains et les fruits, dans le ksar, où, périodiquement, une ou deux fois par semaine, se tient un marché. Dans un rayon de quelques kilomètres autour du ksar campent sous la tente des tribus qui non seulement troquent leur laine et leurs troupeaux contre les produits des sédentaires, mais qui déposent elles-mêmes ou, suivant le terme consacré, ensilotent les grains qu'elles ont pu obtenir par des semailles faites à la volée, au hasard d'une pluie favorable. Leur existence plus ou moins nomade est liée néanmoins à celle de l'oasis. Leurs mouvements gravitent autour d'elle, sans s'en éloigner. Mais ce n'est pas tout. Dans cet échafaudage de relations fondées sur les besoins réciproques, il faut tenir compte aussi d'autres tribus, qui constituent la clientèle lointaine, mais également attirée. On sait qu'en Algérie les tribus voisines du Tell accomplissent périodiquement des migrations vers le Sud pour échanger leurs produits pastoraux contre les dattes dont se compose en partie leur alimentation. Chacune est en rapport avec un ksar particulier où elle est en possession de vendre et d'acheter, en vertu d'un contrat fidèlement observé de part et d'autre. C'est une cause de guerre, si d'autres tribus essayent de la supplanter.

On peut affirmer que ce système de relations est inintelligible sans la connaissance de la physionomie du pays. Elle seule, en nous montrant le mélange de terres arrosées et de terres arides, les nuances intermédiaires qui existent d'un domaine à l'autre, rectifie les conceptions absolues et rétablit la vraie perspective. Et notons bien encore qu'il ne s'agit pas d'un cas isolé. Le mode d'existence que nous venons de décrire repose sur une combinaison qui se répète dans toute la 
zone aride de l'Afrique, comme en Arabie ${ }^{1}$; à la seule différence que c'est tantôt le sédentaire, tantôt le nomade; tantôt l'agriculteur, tantôt le pasteur, et plus souvent celui-ci que celui-là, qui dans cette association se fait la part du maître.

Ce serait le moment de parler de ces oasis, types si curieux euxmêmes d'organisation sociale. La base même de l'édifice social est changée par le fait que l'idée de propriété se transporte e la terre à l'eau, comme il arrive dans les contrées où l'existence d'une vie végétale dépend de l'irrigation. Cette question a déjà été abordée dans ce recueil par M. Jean Brunhes 2; notre collaborateur, par les travaux qu'il poursuit sur cet important sujet, nous donneras sans doute bientôt l'occasion d'y revenir. Contentons-nous de rappeler que ce qu'on appelle les régions arides embrasse, en Amérique comme dans l'ancien continent, en Afrique australe et en Australie comme au Nord de l'Équateur, une étendue dont on ne se faisait pas une idées il y a moins d'un demi-siècle. Quel parti l'homme saura-t-il en tirer ? Comment parviendra-t-il en particulier à utiliser les ressources de l'écoulement souterrain ? La question se pose avec d'autant plus d'urgence que les régions facilement cultivable sont aujourd'hui à peu près entièrement occupées; c'est le nœud de la colonisation de l'avenir.

Prenons un autre exemple, choisi dans les conditions opposées de climat. Il y a dans le Sud-Est de l'Asie des régions de pluie abondante, où périodiquement les fleuves inondent leurs alentours et laissent en se retirant des espaces où l'eau séjourne quelques temps après le retrait des crues. Le riz a été trouvé croissant à l'état naturel dans les parties ainsi submergées. Je crois bien que l'abondance du poisson et la facilité de le recueillir dans les mares abandonnées par les crues, fut la première cause d'attraction qui groupa les hommes dans ces deltas ou ces vallées fluviales. Mais en tout cas la présence de la précieuse

Ceci est bien expliqué par Lady Anne Blunt, A pilgrinage in Nejd, the Craddle of the Arab Race. London, Murray, 1881.

2 J. Brunhes, Les irrigations dans la "région aride " des États-Unis (Ann. de Géog., IV, 1894-1895, p. 12-29). - Les irrigations en Egypte (Ibid., VI, 1897, p. 456-460). - Les grands travaux en cours d'exécution dans la vallée du Nil (Ibid., VIII, 1899, p. 242-251). - La seconde édition de 1' "Egyptian Irrigation » de M. W. Willcox (Ibid., IX, 1900, p. 265-269). 
graminée fut la seconde. On en fît l'éducation; avec quel soin minutieux et quel succès, les nombreuses variétés d'espèces cultivées en témoignent. Ce fut le principe d'une culture qui, par l'abondance de nourriture fournie sur un petit espace, comme par les soins répétés qu'elle exige, a exercé une grande influence sociale. Une famille de cultivateurs de riz, au Cambodge, peut vivre à la rigueur sur un hectare. La propriété est très divisée. Et cependant pour entretenir les parois des rizières, régler la distribution de l'eau, repiquer la plante, la moissonner, l'égrener, la décortiquer, il faut une main-d'œuvre nombreuse et toujours présente. C'est une série d'opérations qui dure plus de six mois; travail menu, d'adresse plus que de force, où la femme a un grand rôle. Ce travail se fait en famille et entre voisins. Le sexe féminin d'un village tout entier se transporte à la ronde chez chaque cultivateur pour procéder rapidement et en temps utile à ces multiples opérations. L'iconographie chinoise ou japonaise nous a familiarisés avec ces scènes. Elles ont trouvés chez quelques-uns de nos résidents européens des observateurs sympathiques ${ }^{3}$. Le cycle traditionnel ramène donc des fêtes ou réjouissances périodiques; il est le cadre dans lequel une foule de petites gens, pullulant entre leurs carrés de rizières et leurs palissades de bambous, mettent leurs joies, leurs superstitions et leurs espoirs.

Je me garderais de trop généraliser; mais s'il est vrai que dans ces sociétés d'Extrême-Orient qui gravitent autour de la Chine, leur centre et leur moteur, la forte constitution de la famille et du village soit la pierre angulaire, on voit le rapport de cause à effet entre le mode de culture inspiré par les conditions géographiques et la seule forme vraiment populaire d'organisation sociale qu'on $y$ découvre. L'importance de ce fait tarda pourtant à être aperçue. Les Anglais se seraient épargné de graves déboires, si, au début de leur domination dans l'Inde, quand ils voulurent organiser le Bengale et le Bahar suivant le principe qui leur était cher de la grande propriété, ils avaient eu un sentiment plus exact des conditions naturelles.

On peut objecter qu'il ne s'agit dans les exemples précédents que de sociétés peu développées ou paraissant figées dans leurs habitudes.

3 Voir : ADHÉMAR LECLÈRE, La culture du riz au Cambodge (Revue scientifique, $4^{\mathrm{e}}$ série, XIII, p. 11-109, passim) 
La civilisation chinoise elle-même garde, en effet, un aspect patriarcal et familial, marqué d'un certain cachet d'archaïsme.

Assurément, le lien est plus délicat à saisir dans nos sociétés extrêmement compliquées. Il n'existe pas moins. Par exemple, l'auteur de l'Anthropogéographie, M. Ratzel, remarque le caractère original que l'étendue des surfaces sur lesquelles opère l'Américain a communiqué à sa civilisation. D'autres observateurs ont insisté également sur ce point de vue. ${ }^{4}$ Il n'y a rien en effet qui déroute davantage l'Européen, et qui s'impose davantage à ses réflexions. L'échelle des proportions n'est pas la même pour eux et pour nous. Nos cadres d'habitudes sont généralement circoncis entre la Méditerranée et la mer du Nord, c'est-à-dire dans un intervalle cinq ou six fois plus petit que celui que l'Américain embrasse entre ses deux Océans. Il convient d'ajouter à l'étendue une autre circonstance non moins importante, c'est la faible densité de population. Si l'on compare aux États-Unis cette province chinoise de Sseu-tch'ouan, une des plus riches mais des plus retirées, où, suivant le rapport de notre Mission Lyonnaise, le salaire journalier moyen d'un ouvrier est d'environ 35 centimes, on a sous les yeux les antipodes du monde économique. Il s'agissait donc pour l'Américain de transporter économiquement à 2 000 kilomètres de distance les produits des Prairies aux ports de l'Atlantique, de rendre mobiles et circulantes des masses énormes de minerais et de houille : c'est par le triomphe du machinisme qu'il y est parvenu. Le développement de la force mécanique sous toutes ses formes, vapeur ou force hydraulique, à tous les degrés, depuis l'élévateur gigantesques jusqu'aux applications les plus minutieuses et délicates, est devenu la marque de l'Américanisme. L'existence d'un outillage incomparable de transport n'a pas été sans influer sur la mentalité américaine. À ces facilités de locomotion se sont adaptées des habitudes de vie qui tranchent avec les nôtres. Les foyers de production et les grand ports dans lesquels leurs produits se centralisent, les pays du blé, du fer, des métaux précieux, les paysages mêmes que l'Américain, fatigué de ses plaines monotones, peut opposer à nos Pyrénées et à nos Alpes, tout se trouve séparé par de grandes distances. Néanmoins ces points éloignés rentrent dans le cercle de son activité,

4 A. OPPEL, Amérique et Américains (Ann. de Géog., VIII, 1899, p. 438-459; IX, 1900, p. 56-64). 
de ses spéculations habituelles; ils se combinent pour lui aussi naturellement que le font pour nous les scéneries qui se concentrent de la Bretagne aux Vosges, de la Flandre à la Côte d'azur. De là des associations d'idées qui rapprochent dans ces esprits des objets pour nous disparates ou très éloignées. Cette disposition les porte, dans les arts, à synthétiser tous les styles. Elle leur inspire, dans leurs rapports avec la vieille Europe, ce remarquable éclectisme qui, malgré le haut sentiment qu'ils ont d'eux-mêmes, les pousse à choisir dans différents pays ce qu'ils jugent de meilleur pour l'incorporer à leur vie nationale. Je laisse à d'autres le soin de dire si finalement ce peuple, mieux préparé que tout autre aux relations à grande distance, ne devait pas aboutir à une politique générale en rapport avec ses habitudes, et à laquelle il ne lui coûterait guère d'accommoder ses visées.

Fin du texte 